\title{
Magnetoelectric Excitations in Hexaferrites Utilizing Solenoid Coil for Sensing Applications
}

\author{
Saba Zare, Hessam Izadkhah, Sivasubramanian Somu and Carmine Vittoria* \\ Department of Electrical and Computer Engineering, Northeastern University, Boston, 02115, U.S.
}

We have developed techniques for $\mathrm{H}$ - and E-field sensors utilizing single phase magnetoelectric hexaferrite materials in the frequency range of $100 \mathrm{~Hz}$ to $10 \mathrm{MHz}$. Novel excitation method incorporating solenoid coils and single and multi-capacitor banks were developed and tested for sensor detections. For H-field sensing we obtained sensitivity of about $3000 \mathrm{~V} / \mathrm{mG}$ and for E-field sensing the sensitivity was $10^{-4} \mathrm{G} / \mathrm{Vm}^{-1}$. Tunability of about $0.1 \%$ was achieved for tunable inductor applications. However, the proposed designs lend themselves to significant $\left(\sim 10^{6}\right)$ improvements in sensitivity and tunability.

\section{INTRODUCTION}

Recently, single phase magnetoelectric (ME) hexaferrites [1-4] have exhibited high ME coupling, $\alpha$, at room temperature. As such, there appears to be potential to develop and fabricate novel devices based on the ME effect. The ME effect implies that the application of a magnetic field, $\mathbf{H}$, induces an electric Polarization, $\mathbf{P}$, and in the converse case applying an electric field, $\mathbf{E}$, magnetization, $\mathbf{M}$, in the material is induced. The magnetoelectric coefficient, $\alpha$, defines the relation between $\mathbf{P}$ and $\mathbf{H}(\mathrm{P}=\alpha \mathrm{H})$ as well as $\mathbf{M}$ and $\mathbf{E}(\mathrm{M}=\alpha \mathrm{E})$ in the converse one with unit of Sec./m for both cases. Although single phase ME materials operating at room temperature have been a recent revelation, composite materials consisting of magnetostriction and piezoelectric layers mechanically attached to each other have been used in device developments for much longer time [5-7]. The ME effect in composites structures implies that the application of an electric field, $\mathbf{E}$, induces a change in magnetostrictive internal field, $H_{\lambda}$, in the magnetostrictive layer and an application of $\mathrm{H}$ induces a change in $\mathbf{P}$ or $\mathbf{E}$ in the piezoelectric layer. The two layers are coupled to each other via the strain across the interface. Several types of devices have been made using ME composite structures including tunable filters [8] and inductors [9] by applying a DC electric field to a piezoelectric slab. Magnetic field sensors have been developed utilizing mechanical resonance of piezoelectric/magnetostriction cantilever configurations [10] by strain changing the piezoelectric layer. However, coupling the piezoelectric layer effectively onto a metallic magnetostrictive layer through incorporating of piezoelectric slab into magnetic devices or deposition of magnetic layers on piezoelectric material may be complicated in fabrication and sometimes challenging for integration purpose.

*Corresponding author: c.vittoria@neu.edu.

(C) 2015. This manuscript version is made available under the Elsevier user license http://www.elsevier.com/open-access/userlicense/1.0/ 
Evolution of single phase ME hexaferrites make it possible to have cross coupling between electric and magnetic fields in a single layered structure and, thus, it is time to look into the next generation of devices as sensors or tunable microwave devices. Again, strain is the mediator for the coupling between fields, but it is generated within the single ME hexaferrite layer. So far, experiments on ME hexaferrites have been performed at very low frequencies ( DC) to examine polarization measurements [11] and at high frequencies [12] in the microwave regime (GHz ranges). In this paper, we explore the ME effect in single phase hexaferrites in the range of $100 \mathrm{~Hz}$ to $10 \mathrm{MHZ}$ for the purpose of investigating potential applications for magnetic field and electric field sensors or any tunable devices in this range of frequencies. Potential applications include biomedical sensing, for example, in magnetoencephalography (MEG) [13] and magnetocardiography (MCG) [14]. Two types of experimental techniques have been designed and implemented for testing. In the first technique, we refer to it as a "direct" method, an ac magnetic field, $\mathbf{H}$, generated via a solenoid coil, induces an electric field, $\mathbf{E}$, or polarization, $\mathbf{P}$. These induced fields are measured in a capacitor detection scheme. The second technique, referred to as the "converse" method, an ac voltage or electric field, $\mathbf{E}$, is applied via a capacitor and the induced magnetization, $\mathbf{M}$, is measured. The induced magnetization is detected via a solenoid coil utilizing Faraday's law. In these two sets of experiments the emphasis has been to develop techniques to probe the ME effect in single phase materials for potential sensor detectors.

\section{Experimental Apparatus}

A bulk single crystal Z-type hexaferrite sample, $\mathrm{Sr}_{3} \mathrm{Co}_{2} \mathrm{Fe}_{24} \mathrm{O}_{41}$ was utilized in all of our sensor circuits. The ME single phase material was characterized in previous vibrating sample magnetometer (VSM) experiments yielding a $\alpha$ value of $10^{-6} \mathrm{Sec} . / \mathrm{m}[15]$. Since the sample shape was that of a slab, the E field was applied normal to the slab plane in order to reduce voltage requirements in generating E fields as in a converse ME experiment, for example. Besides applying E normal to the slab plane, we have developed a technique to apply $\mathrm{E}$ in the plane of the slab and still maintain voltage requirements relatively low to induce ME effects in devices, Fig. 1. For this purpose parallel metallic strips were deposited on the plane of the slab. Thus, detection (direct) or application (converse) of E may simply be accomplished using a single capacitor, Fig. 1a, or multi-capacitors, Fig. 1b, arrangements. In the single capacitor arrangement electrodes are deposited across both surfaces of the slab. There are five capacitors to generate or detect $\mathrm{E}$ in the plane of the slab. The slab thickness was $0.5 \mathrm{~mm}$ and the lateral dimensions were 5 and $6 \mathrm{~mm}$. The 
width of the conductive strips was $0.5 \mathrm{~mm}$ and the gap between strips $0.5 \mathrm{~mm}$. A solenoid coil was used to detect (converse) or apply an $\mathrm{H}$-field (direct). The diameter of the solenoid was $10 \mathrm{~mm}$, the length $16 \mathrm{~mm}$ and the number of turns 500 .
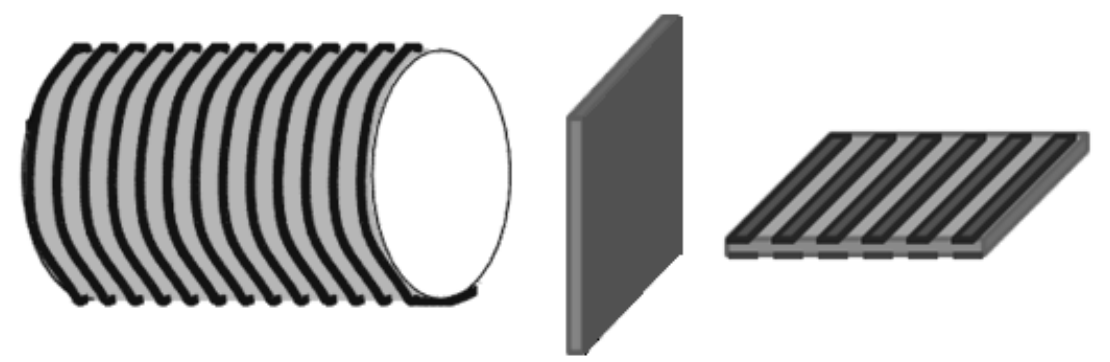

Fig. 1 Solenoid and two cases of capacitor arrangements: a) single capacitor and b) multi-capacitors.

\section{Direct ME Method- H Field Sensor}

An ac voltage was applied to a solenoid coil (inductance value of $5.4 \mathrm{mH}$ ) in order to generate an ac magnetic field, $\mathbf{H}$, to the ME sample. The induced electric field, $\mathbf{E}$, in the ME sample was detected either using a single capacitor circuit (E perpendicular to the slab plane) or multi-capacitors circuit (E in the slab plane), see Figs. 1-2. Thus, ac current flowing through either capacitors arrangements monitored the polarization, $\mathbf{P}$, and, therefore, $\mathbf{E}$, see Eqs. 1-4.

\section{Single Capacitor Detection}

In the circuit shown in Fig. 2 the capacitor was electrically biased to $\mathrm{E}_{b}=180 \mathrm{~V} / \mathrm{m}$ via the $20 \mu \mathrm{F}$ capacitor. A $\mathrm{DC}$ voltage of $0.11 \mathrm{~V}$ was applied to the capacitor $\left(\mathrm{V}_{b}=0.2 \mathrm{~V}\right)$. Also, we poled or biased the ME material in static magnetic fields, $\mathrm{H}_{b}$, of 1800 and 550 Oe. $\mathrm{H}_{b}$ was applied perpendicular to the ac magnetic field generated in the solenoid coil, $h_{e x}$. The object of the biasing fields was to remove residual domains and prepare sample into single magnetic and electric polarized domains. The induced polarization, $\mathbf{P}$, was measured by monitoring the current

through the resistor, Ro, wher $\mathrm{P}_{d}=\frac{I o / j \omega}{A}$. A is the cross area of capacitor inserted within the solenoid coil and $\omega$ is the radial frequency. The measured peak to peak polarization, $\mathrm{P}_{d}$, for single capacitor detection is to be compared 
with multi-capacitors detection in later in the text for two values of poling magnetic fields of the sample. The electric susceptibility, $\chi_{\boldsymbol{e}}$, of the sample was obtained from the relationship that $P_{d}=\varepsilon_{0} \chi_{\boldsymbol{e}} \frac{v_{d}}{\mathrm{~d}}$, where $v_{d}$ is the peak to peak voltage across the capacitor and $d$ is the thickness of the slab or capacitor width. We measured $\chi_{\boldsymbol{e}}$ to be 7020. Excited or applied and biased are labeled with subscripts "ex" and "b", respectively. Fields that are detected by the subscript "d". It is noted that for single capacitor detection circuit all the fields are parallel to each other and perpendicular to the slab plane(C-axis), except for the biasing or poling magnetic fields applied in the slab plane, see Fig. 2.

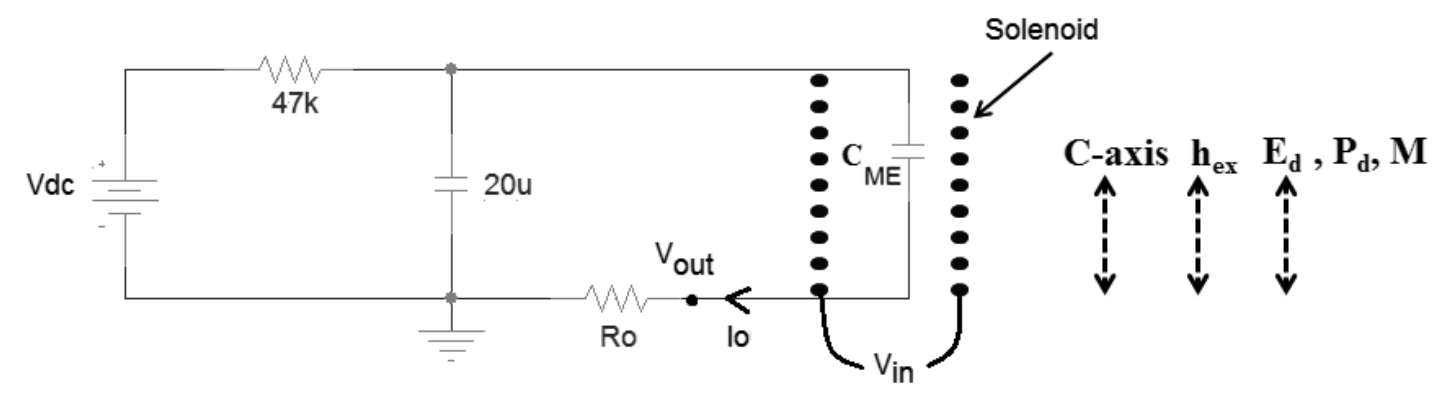

Fig.2. Circuit for single capacitor detection in a Direct ME experiment. At the right side directions of the C-axis of material, $\mathrm{h}_{\mathrm{ex}}, \mathrm{E}, \mathrm{P}$ and $\mathrm{M}$ is depicted; all are in the same direction.

However, it is pointed out that $h_{e x}$ represents an indirect field of excitation, since the basic interaction or coupling is between internal magnetic excitation fields like $\mathrm{h}_{\mathrm{i}}$ and internal electric fields. In general $h_{e x} \neq h_{i}$, especially for slab shaped samples. In particular, the internal magnetic ac field, $\mathbf{h}_{\mathbf{i}}$, in the ME sample perpendicular to the slab plane is significantly reduced from $h_{e x}$ due to the demagnetizing field. The relationship between $h_{i}$ and $\mathrm{h}_{\mathrm{ex}}$ is given [16] as

$$
h_{i}=\left(h_{e x}-N_{\perp} m_{i}\right)
$$

where $N_{\perp}$ is the demagnetizing factor perpendicular to the plane of the ME slab, and $m_{i}$ is the internal ac magnetization, $m_{i}=\chi_{m} h_{i} \cdot \chi_{m}$ is the magnetic susceptibility. The demagnetizing factor may be approximated as follows [16]

$$
N_{\perp}=4 \pi \frac{1}{\left(1++^{C} /\left(\frac{a b}{a+b}\right)\right.},
$$

where $\mathrm{a}, \mathrm{b}$, and $\mathrm{c}$ are dimensions of the slab, $\mathrm{a}$ and $\mathrm{b}$ in the plane and $\mathrm{c}$ perpendicular to the plane of slab. 
Hence,

$$
h_{i}=h_{\text {ext }} /\left(1+N_{\perp} \chi_{m}\right)
$$

The ME effect relates the internal ac fields $h_{i}$ to $\mathrm{P}_{d}$ as follows

$$
P_{d}=\alpha_{\|} h_{i}=\alpha_{\|} h_{e x t} /\left(1+N_{\perp} \chi_{m}\right)
$$

where $\alpha_{\|}=2.32 * 10^{-6} \mathrm{sec} / \mathrm{m}[15]$.

We find that the magnetic susceptibility, $\chi_{m}$, is in the order of $1 / 4 \pi$ consistent with the susceptibility of a non-saturated magnetic state at low frequencies. Fig. 3 shows the internal magnetic field normalized with respect to the external exciting magnetic field, $\mathrm{h}_{\mathrm{ex}}$.

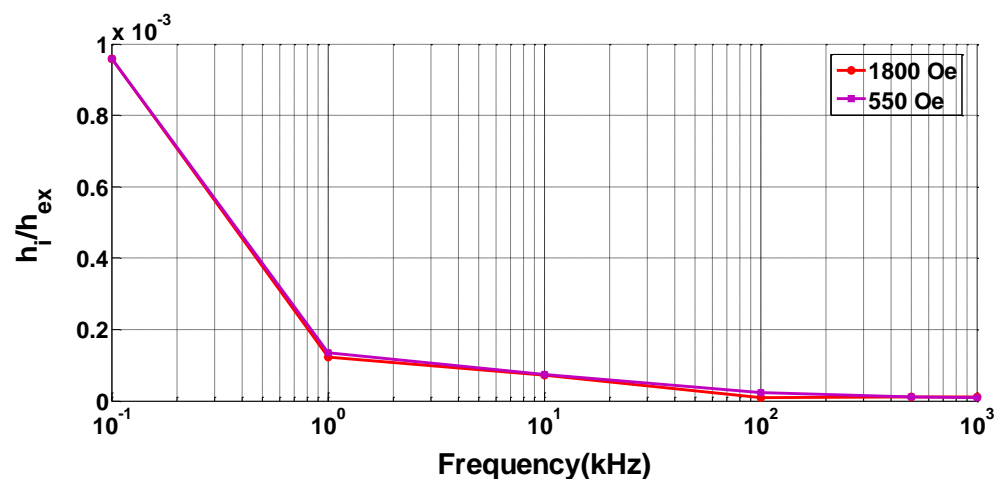

Fig. 3. Normalized internal field with respect to the external exciting field.

We define sensitivity as the ratio of the measured or detected induced electric field across the single capacitor with respect to the internal magnetic field, $h_{i}$, as $S=E_{d} / h_{i}$. Measured $\mathrm{S}$ for a single capacitor detector is to be compared to $\mathrm{S}$ measured using multi-capacitors detectors, although definition of $\mathrm{S}$ is $\mathrm{E}_{\mathrm{d}} / \mathrm{h}_{\mathrm{ex}}$ for multi-capacitors case. As we shall see the demagnetizing fields for the latter case are minimal.

\section{Multi-Capacitors Detection}

In the multi-capacitors detection of $\mathrm{P}_{d}$ and/or $\mathrm{E}_{d}$ in the plane of the slab, there are several conductive strip lines (six lines in our device) on the slab plane parallel to each other (see Fig. 1) with a distance of $0.5 \mathrm{~mm}$ between two successive strips. The sample was poled or magnetically biased, $H_{b}$, by two different dc magnetic fields of 1800 and 550 Oe perpendicular to $\mathrm{h}_{\mathrm{ex}}$. The axis of the solenoid was parallel to $h_{e x}$ and in the plane of the slab. $H_{b}$ 
was perpendicular to $h_{e x}$, but also in the plane of the slab. The bias de voltage of $3 \mathrm{~V}$ was applied to a resistor bank so that each capacitor was equally biased. The spacing between capacitors and the resistor across each capacitor were designed to be the same for all the capacitors so that the detected ac voltage appearing across each capacitor was the same. Fig. 4 shows the voltage divider (detecting circuit) and the equivalent circuit model of the multicapacitors detection method.
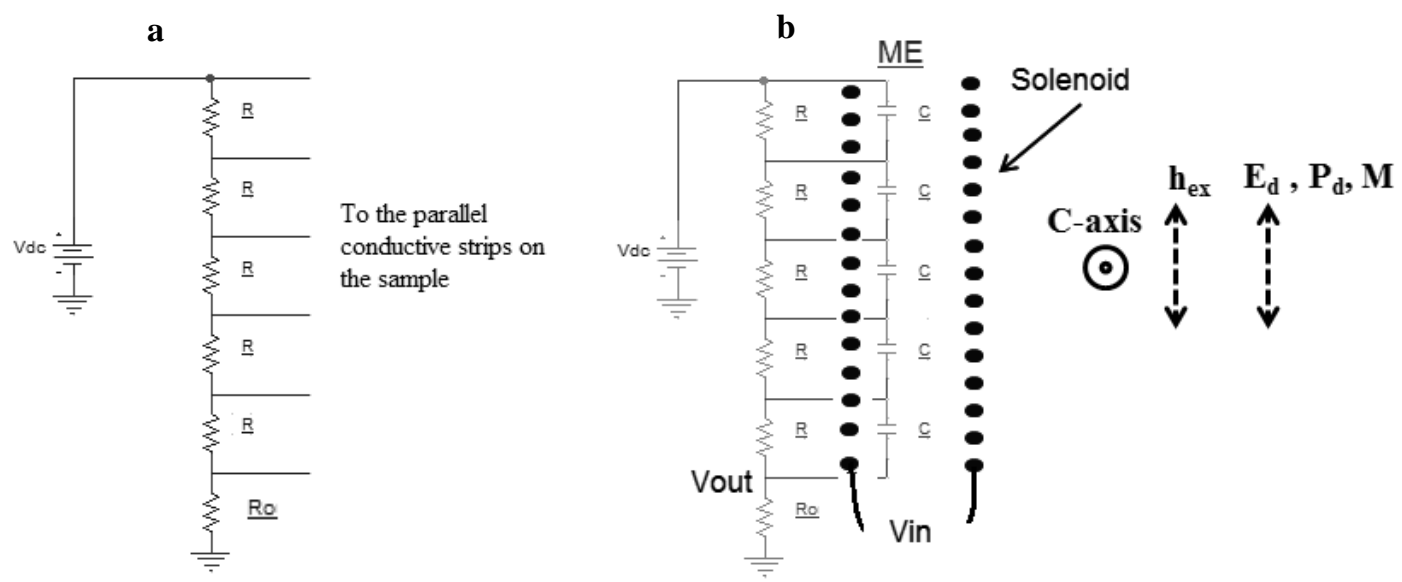

Fig. 4 Voltage divider (detecting circuit) and the equivalent circuit model of the parallel strips configuration after connecting to the conductive lines in the plane of the $\mathrm{ME}$ sample with directions of the $\mathrm{C}$-axis of material, $\mathrm{h}_{\mathrm{ex}}, \mathrm{E}, \mathrm{P}$ and $\mathrm{M}$. $\mathrm{R}=8.9 \mathrm{~K} \Omega$.

Since the detected polarization $\mathrm{P}_{d}$ is proportional to current flow only across $\mathrm{C}$ and not $\mathrm{R}$, we needed to determine the voltage across $\mathrm{C}$ and the value of $\mathrm{C}$. In order to obtain the value of $\mathrm{C}$ we plotted $\mathrm{V}_{\text {out }} / \mathrm{V}_{\text {in }}$ versus frequency, see Figs.4-5, in which $V_{\text {out }}$ is the output voltage across Ro and $V_{\text {in }}$ in the ac voltage applied across the coil. We may consider this gain graph, Fig. 5 , as a Bode plot with a zero at $1 \mathrm{MHZ}$ in which $1 / \mathrm{R}_{O}=\omega \mathrm{C}$, where $\mathrm{R}_{O}=$ 8.9 K $\Omega$. C was calculated to be $\mathrm{C}=17 \mathrm{pF}$.

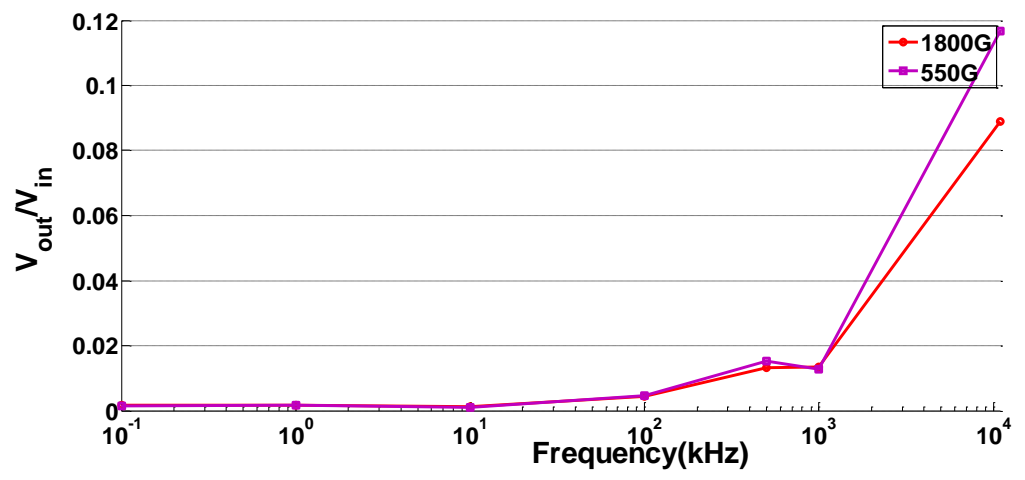

Fig. 5 Gain of the detector circuit. The ratio of $\mathrm{V}_{\text {out }} / \mathrm{V}_{\text {in }}$ is plotted as a function of frequency. The voltage detected across each capacitor, $\mathrm{V}_{\mathrm{C}}$, and $\mathrm{C}$ are determined from this plot. 
The basic difference between the single capacitor detection technique proposed in the previous section and the multi-capacitors parallel strips detection is that the detected polarization, $\mathrm{P}_{d}$, and/or electric field, $\mathrm{E}_{d}$, induced by an applied ac magnetic field, $\mathrm{h}_{\mathrm{ex}}$, are directed in the slab plane for the multi-capacitor case, but perpendicular to the slab plane for the single capacitor case. For either case $\mathrm{h}_{e x}$ is parallel to $\mathrm{E}_{d}$ or $\mathrm{P}_{d}$. It is noted that the $\mathrm{C}$-axis is perpendicular to the slab plane. As in previous section the detected polarization $\mathrm{P}$ may be determined from either $P_{d}=\frac{I c / j \omega}{A}$ or $P_{d}=\varepsilon_{0} \chi_{e} \frac{V c}{\mathrm{~d}}$, where $\mathrm{V}_{\mathrm{C}}$ is the voltage across each capacitor and $\mathrm{I}_{\mathrm{C}}$ the current flow through each capacitor. However, very careful interpretation of $\mathrm{A}, \mathrm{d}$, and $\chi_{e}$ needs to be exercised here. In previous section for a single capacitor those interpretations are conventional and simple. For multiple capacitors as we have in this section, it is not so simple. We have opted for the following approximations: $\mathrm{d}=1 / 2 \mathrm{~mm}$, and $\chi_{e}=3500$. The average value of $\chi_{\boldsymbol{e}}$ includes the value of $\chi_{\boldsymbol{e}}$ deduced in previous section and that of free space. The value of $\mathrm{d}$ is estimated from the separation of the electrodes. In Fig. 6 we plot $\mathrm{P}_{d}$ as induced by $\mathrm{h}_{\mathrm{ex}}$ in a direct ME method utilizing multiple capacitors or metallic strips as well as for the single capacitor detections.

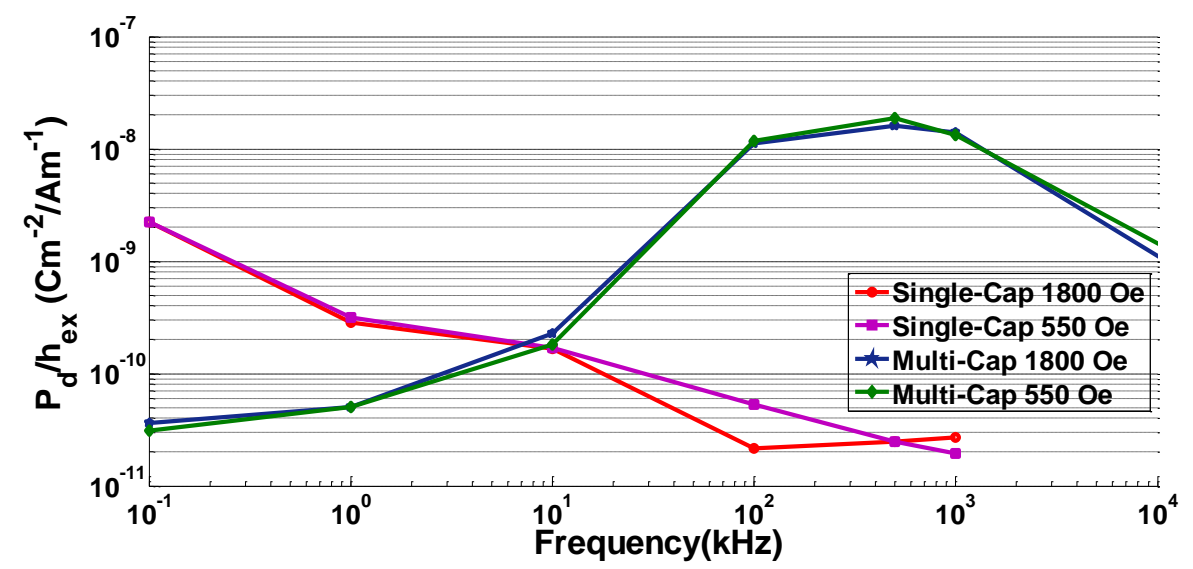

Fig. 6. Polarization induced in the ME sample vs. frequency which is normalized by the excited or applied ac magnetic field.

For single capacitor detection $P_{d}$ decrease with frequency, as $\chi_{m}$ is proportional with frequency for frequencies well below ferromagnetic resonance (FMR), see Eq. 3. For the multi-capacitors case the induced polarization exhibits a maximum near $\sim 800 \mathrm{kHz}$. In addition, the direction of electric field measured across each successive strips $\left(\mathrm{E}_{d}=\mathrm{Vc} / \mathrm{d}\right)$ and $\mathrm{h}_{\mathrm{ex}}$ generated in the solenoid are parallel to each other and both are directed in the 
plane of the slab, perpendicular $(\perp)$ to the $\mathrm{C}$ axis. Hence, one can deduce the ME coupling factor from $P_{d}=$ $\alpha_{\perp} h_{e x}$. We have assumed that $h_{e x} \approx h_{i}$, since demagnetizing factor is relatively small (see discussion in previous section). We obtained $\alpha_{\perp}$ to be in the order of $10^{-9}<\alpha_{\perp}<2 * 10^{-8} \mathrm{sec} / \mathrm{m}$. We conclude that $\alpha_{\perp}$ is smaller than $\alpha_{\|}$ which is not surprising, since the ME coupling is anisotropic in single crystal ME hexaferrites. The peak value in $\mathrm{P}_{\mathrm{d}}$ may be attributed to acoustic standing mode resonance. For example, if we assume an acoustic velocity of $\sim$ $5 \times 10^{5} \mathrm{~cm} / \mathrm{s}$, the half-wavelength excitation would be $\sim 3 \mathrm{~mm}$ corresponding to the dimensionality of the device.

Finally, the $\mathrm{H}$ field sensitivity, $\mathrm{S}$, of the multiple capacitors detection or multi-strips detection is defined (as before for the single capacitor case) as the ratio of the detected electric field in the plane of the sample over the internal magnetic field, see Fig. 7.

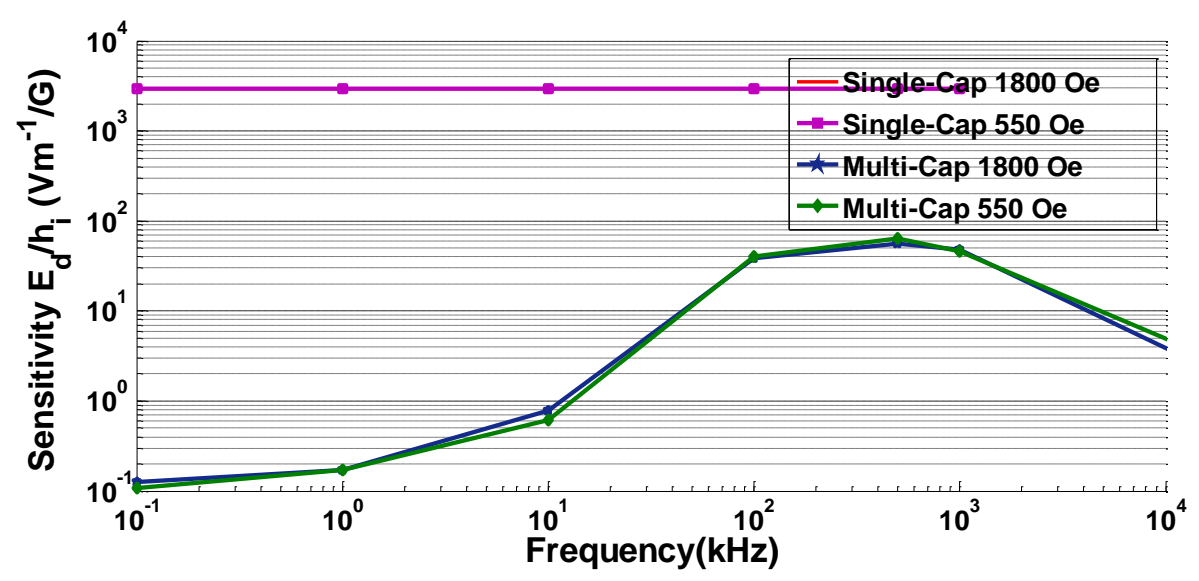

Fig. 7. Sensitivity vs. frequency for both types of detection methods.

The smaller sensitivity measured in this detection scheme compared to the single capacitor detection is due to the smaller ME coupling coefficient. It is noted that by narrowing the gap spacing between strips the detected electric field can be enhanced as much as a factor of 1000 and, therefore, increase sensitivity by that factor. As in Fig. 6, a maximum in sensitivity was observed using multi-capacitors detection at $\sim 800 \mathrm{kHz}$. This implies that $\mathrm{S}$ scales as the induced polarization, as one would expect.

\section{Converse ME Method- E Field Sensor}

In the Converse ME method, an ac voltage or electric field, E, is applied or excited to the ME sample via a single capacitor or multi-capacitors and the change in magnetization, $\mathrm{M}$, is detected via a voltage on a solenoid coil (Faraday's law). In the single capacitor excitation the induced magnetization or flux direction is perpendicular to the 
slab plane. In the multi-capacitor mode excitation the induced magnetization or flux is assumed to be in the slab plane. The voltage on the coil is induced by a change in magnetic flux generated within the ME sample. The sample "radiates" magnetic flux to be detected up by the coil. The detection circuit schematic is shown in Fig. 8 .

\section{Single Capacitor Excitations}

An ac voltage of $0.6 \mathrm{Vp}-\mathrm{p}$ is applied or excited across the capacitor, C, see Fig. 8. The peak to peak excited electric field, $\mathrm{E}_{\mathrm{ex}}$, was $1 \mathrm{KV} / \mathrm{m}$. Also, $H_{b}=120$ Oe was applied parallel to $\mathrm{E}_{\mathrm{ex}}$, perpendicular to the slab plane. The purpose of $\mathrm{H}_{b}$ is to prepare sample into a single magnetic domain.

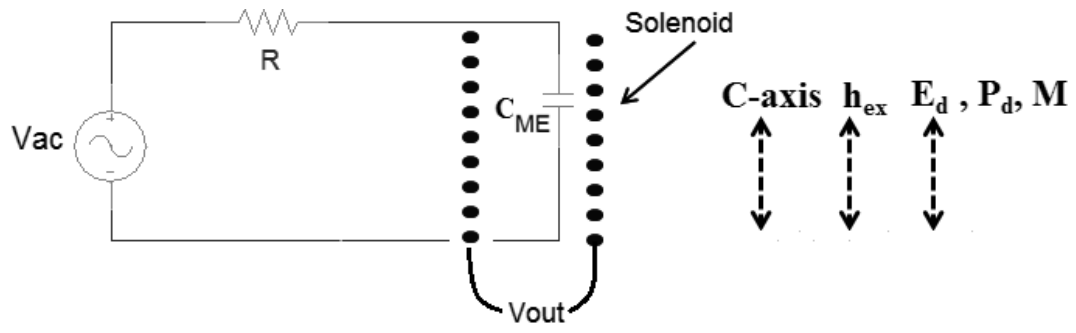

Fig. 8 Circuit used for the converse ME method utilizing a single capacitor excitation, $R=22 \Omega$.

As it is described above the voltage was detected in the coil through the flux generated within the sample. The ME effect allows for changes in $M$ due to $\mathrm{E}_{\mathrm{ex}}$. The change in magnetization due to the $\mathrm{ME}$ effect induces "magnetic" change at the surface of the sample (Gauss' Law). Much like electric charges, magnetic "charges" radiate flux lines that are detected by the coil $\mathrm{N}$ times. The peak to peak voltage, $\mathrm{V}_{\text {out }}\left(\mathrm{V}_{\mathrm{t}}\right)$, was measured or detected on the coil due to Faraday's law and considered as the detected output signal, Eq.6.

$$
\Phi_{t}=\frac{V_{t}}{2 \pi f}
$$

where $\mathrm{f}$ is the frequency and $\Phi_{t}$ is the total magnetic flux detected by the solenoid coil. Basic question to consider in this paper is: how effectively is the flux generated by the sample, $\Phi_{S}$,

$$
\Phi_{S}=B * A=\alpha_{\|} * E_{e x} * A
$$

detected by the coil. We define the coupling as the portion of the flux detected by the coil, $\Phi_{D}$, 


$$
\Phi_{D}=\frac{\Phi_{t}}{N}
$$

divided by the maximum flux generated by the ME sample.

$$
\text { Coupling }=\frac{\Phi_{D}}{\Phi_{S}}
$$

It is obvious that the more compact the sample and the pick-up coil are put together in the circuit, the better the coupling and sensitivity can be.

\section{Multi-Capacitors Excitations}

The converse method for multi-capacitors excitations is very similar to the single capacitor excitation method (Fig. 8). An ac voltage of $1.2 \mathrm{~V}$ (peak to peak) was applied to a voltage divider resistors bank, similar to Fig. 4 without the insertion of Ro. A bias magnetic field of 150 Gauss was applied in the plane of the slab sample. The exciting and detected fields are both in the slab plane and parallel to each other. Again, the change in magnetization in the sample is detected via the solenoid coil. The coupling factor for this configuration is calculated as before (see

Eq. 9). Fig. 9 shows the measured coupling factor vs. frequency for the solenoid coil pick- up for both the single capacitor and multi-capacitors excitations.

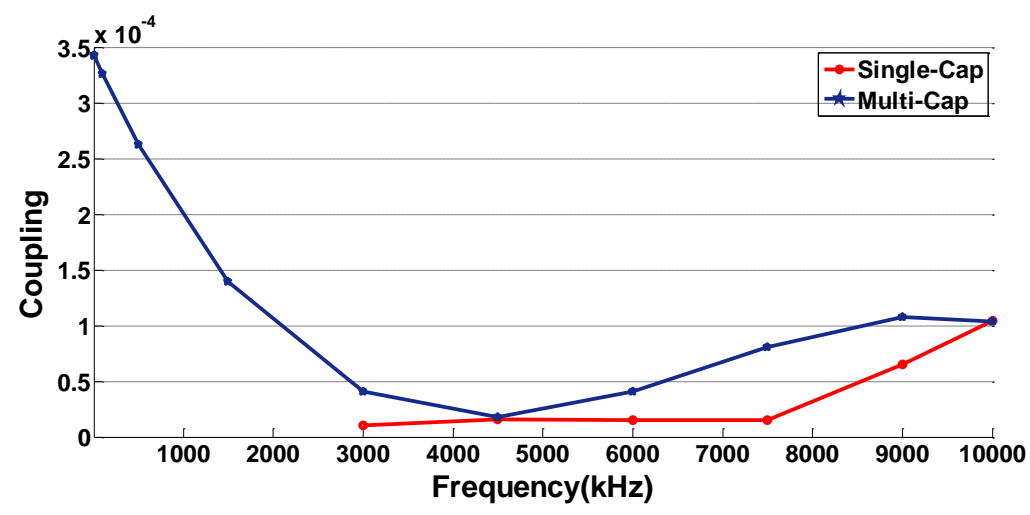

Fig. 9. The coupling factor defined as $\frac{\boldsymbol{\Phi}_{D}}{\boldsymbol{\Phi}_{S}}$, see text.

The measured values of coupling are well below the ideal value of 1 . However, once again, the multi-capacitor excitation gave rise to an extremum point or minimum at $\sim 4.5 \mathrm{MHz}$. Clearly, future circuit designs must allow for physical contact between ME samples and detection coils, if coupling is to be increased. In Fig. 10 the ratio of coil 
output voltage, $\mathrm{V}_{t}$, divided by $\mathrm{V}_{\mathrm{ex}}$ is plotted as a function of frequency, where $\mathrm{V}_{\mathrm{ex}}$ is the applied or the excitation ac voltage to the single or multi-capacitors. In some sense this ratio may be viewed as a gain voltage ratio, G. It is remarkable that $\mathrm{G}$ is near unity, although the coupling is much less than ideal and the turns ratio is only about $\mathrm{N}=$ 500. Indeed it may be possible to exceed unity gain at reasonably high frequencies by increasing $\mathrm{N}$ or improving the coupling. The frequency dependence of $\mathrm{G}$ is simply a reflection of Faraday's law which states that the output voltage scales with frequency. It is noted that by reducing the gap between two successive conductive strips or electrodes $\mathrm{G}$ will increase as the electric field is inversely proportional to the strips separation and this may be an advantage over the single capacitor shape configuration.

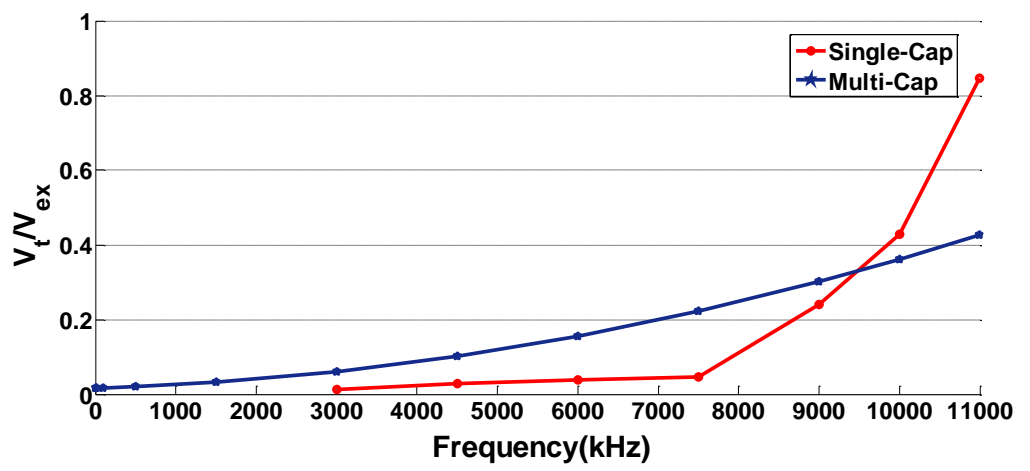

Fig. 10. The gain of the sensor, output voltage on the solenoid coil divided by the applied voltage on each small capacitor between two electrodes.

The sensitivity of the detector is defined as the magnetic flux density field, $\mathrm{B}_{d}$, detected by the coil $\left(\Phi_{t} / A\right.$, $A=$ area of coil) divided by the applied or excitation electric field across the single capacitor or multi-capacitors, $\mathrm{S}=\mathrm{B}{ }_{d} / \mathrm{E}_{\mathrm{ex}}$ in units of $\mathrm{G} / \mathrm{Vm}^{-1}$, see Fig. 11.

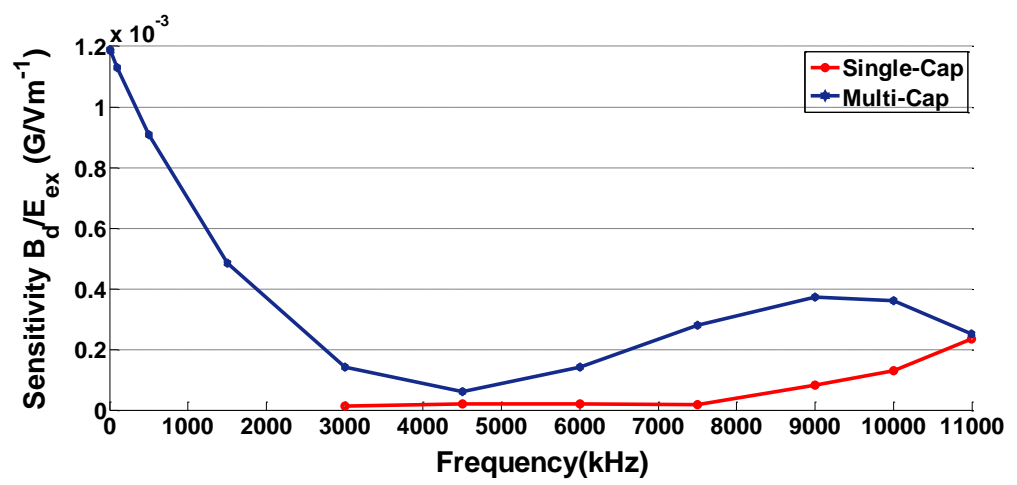

Fig. 11. Sensitivity of converse experiments utilizing both excitation arrangements, see text. 
As expected there appears an interesting correlation between coupling and $\mathrm{S}$ in that both exhibit a minimum at 4.5 MHz for the multi-capacitor excitations. The common factor in both measurements is that excitations were via an interference structure like the multi-capacitors circuit. Finally, if we consider the solenoid coil as an inductor the inductance of the coil can be tuned by applying the excitation voltage, $\mathrm{V}_{e x}$, both across the single capacitor and the solenoid coil. We define tunability as the ratio of the total flux detected by the coil, $\Phi_{t}$, divided by the flux generated by the solenoid coil if driven by $\mathrm{V}_{e x}$. The flux generated by the coil in the absence of ME material is Lxi, and $\mathrm{i}$ is the current generated by $\mathrm{V}_{e x}$, for example. In Fig. 12 tunability was plotted as a function of frequency.

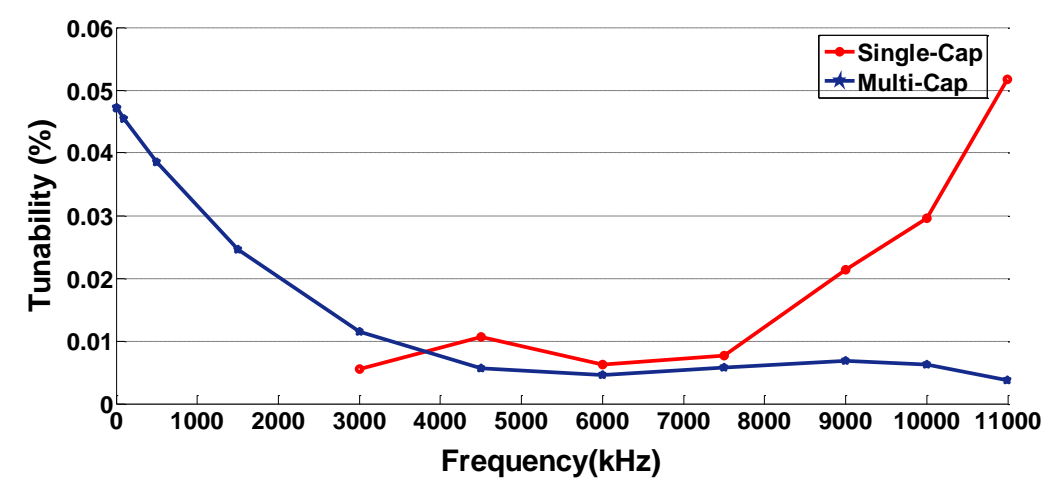

Fig. 12 Tunability of the solenoid coil as an inductor by applying voltage across the parallel strips and coil.

A shallow minimum appears again for the case of multi-capacitors excitation. Clearly, tunability depends strongly on the coupling factor for tunable device applications, as tunability scales as the coupling factor, see Fig. 9.

\section{Non-linear Excitations}

In the converse experiments whereby we applied simultaneously an ac voltage to a single capacitor or multicapacitors and an ac current to the solenoid coil at the same frequency, we have observed at the solenoid output voltage at higher harmonic excitations whose amplitude depended on the voltage drive, see Fig. 13. In Fig. 13 the drive or fundamental frequency was $5 \mathrm{MHz}$, but higher harmonic signals were measured at 10 and $15 \mathrm{MHz}$. At higher frequencies the signals were too weak to detect. The output signal voltage at the fundamental frequency was due to the voltage drive at the capacitors side as well as at the coil. The output signals at higher harmonics was due to the fact that the total current in the solenoid coil was out of phase with the total flux generated in the solenoid 
coil. Clearly, the current contribution arising from the ME effect resulting from voltage applied to the capacitors must necessarily be out of plase with the direct current drive to the coil. Therefore, the resultant inductance was necessarily time dependent implying that voltage resulting from time dependence inuctance will contain higher harmonic components at the coil output.

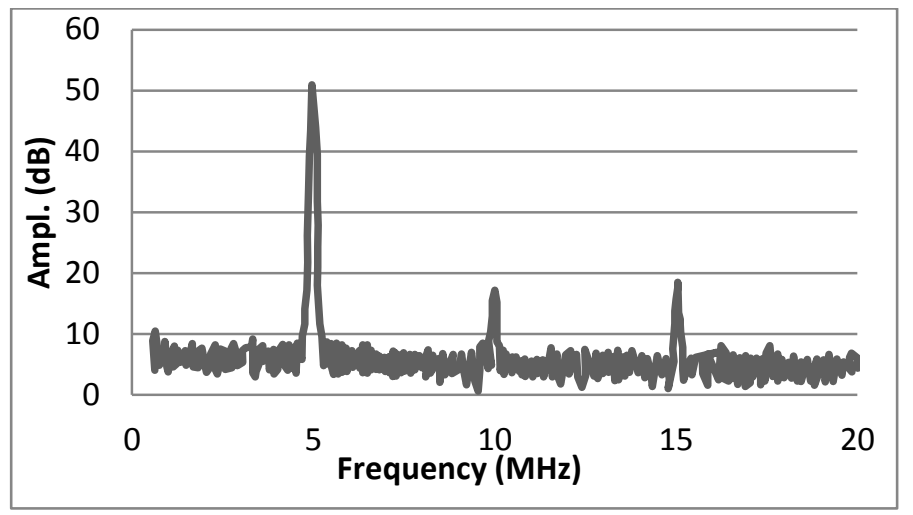

Fig. 13. The fundamental frequency was excited at both sites (capacitor and coil inputs). The harmonic excitations occurred at 10 $\mathrm{MHz}$ and $15 \mathrm{MHz}$ and their amplitude depended on the voltage drive at capacitor terminals.

\section{Discussions and conclusions}

In this paper we developed two techniques to detect $\mathrm{H}$ or E-fields from 100 to $10 \mathrm{MHz}$ in novel circuits utilizing ME hexaferrite materials. We utilized two kinds of circuit configurations: 1. E- or H-field were applied or sensed perpendicular to the ME slab sample. A single capacitor was used for this purpose. 2. E- or H-field were applied or sensed in the plane of the ME slab sample. A multi-capacitors arrangement was used in the circuit.

In the direct ME methods, where the applied ac magnetic field was sensed by method 1 the sensitivity was measured to be about $3000 \mathrm{~V} / \mathrm{mG}$ for the whole range of frequencies tested. In method 2 whereby parallel strips detection were used the sensitivity was 0.1 to $100 \mathrm{~V} / \mathrm{mG}$. The lower sensitivity of the parallel strips configuration arises from the fact that the ME coupling coefficient is smaller when measured in the plane of the sample. We note that in parallel strips technique the gap between parallel lines can easily be made to be closer to each other (for our experiment was about $1 / 2 \mathrm{~mm}$ ) by a factor of 1000 times enhancing the sensitivity. Improved value of sensitivity may be sufficient to detect magnetic fields as small as pico Tesla at $100 \mathrm{~Hz}$ for MEG or MCG applications.

In the converse ME methods the purpose was to examine the feasibility of E-field sensing and/or tunability of a magnetic coil. In our circuit design the coil coupling was measured to be $10^{-5}$ to $10^{-4}$ for the single capacitor excitation configuration and about $10^{-4}$ to $10^{-3}$ for multi-capacitors excitation. For the first time practical E fields 
were applied in the plane of a ME slab material whereby dimensions are rather large. Thus, multi-capacitor excitations may allow for integrated circuits. Sensitivity was measured to be about $10^{-4} \mathrm{G} / \mathrm{Vm}^{-1}$. For the parallel strips configuration the sensitivity reached a value of $10^{-3} \mathrm{G} / \mathrm{Vm}^{-1}$. The voltage gain $\mathrm{G}$ was measured for the single capacitor excitation to be 0.01 to 0.8 . $\mathrm{G}$ was of the same order of magnitude for multi-capacitor excitations. It is remarkable that there is a strong possibility of obtaining $\mathrm{G}$ values greater than unity with simple re-designing of spacing between electrodes or increasing the number of turns in the coil. Tunability of about $0.05 \%$ for the solenoid coil was measured for single capacitor excitation and about the same for parallel strips configuration. Improvements in coupling is reasonable to be expected and, therefore, in tunability. The mechanism for non-linear excitations requires simultaneous excitations at the capacitor circuits as well as at magnetic coil site or circuit. Clearly, parasitic capacitors pick-ups cannot explain the nonlinear response of this device. Both the current drive across the coil and the current contribution arising from the ME effect resulting from voltage applied to the capacitors are responsible for fundamental frequencies at output voltage across the coil. Increased coupling would indeed enhance non-linear excitations as observed in this paper.

\section{Acknowledgements}

We wish to acknowledge and thank the support of the NSF, DMR 1002543 and ECCS 1405108.

\section{References}

${ }^{1}$ Y. Kitagawa, Y. Hiraoka, T. Honda, T. Ishikura, H. Nakamura, and T. Kimura, Nature Mater. 9, 797 (2010).

${ }^{2}$ L. Wang, D. Wang, Q. Cao, Y. Zheng, H. Xuan, J. Gao, and Y. Du, Sci. Rep. 2, 223 (2012).

${ }^{3}$ K. Ebnabbasi, C. Vittoria, and A.Widom, Phys. Rev. B 86, 024430 (2012).

${ }^{4}$ K. Ebnabbasi, M. Mohebbi, and C. Vittoria, J. Appl. Phys. 113, 17 C703 (2013).

5 C.W. Nan, M. I. Bichurin, S. Dong, D. Viehland and G. Srinivasan, J. Appl. Phys. 103, 031101 (2008).

${ }^{6}$ M. Fiebig, J. Phys. D: Appl. Phys. 38, R123 (2005).

${ }^{7}$ C.S. Park, D. Avirovik, M. I. Bichurin, V. M. Petrov, S. Priya, Appl. Phys. Lett. 100, 212901 (2012).

${ }^{8}$ A. S. Tatarenko, V. Gheevarughese, G. Srinivasan,

O. V. Antonenkov, M. I. Bichurin, J. of Electroceramic, 24, 5 (2007)

9 Y. Gao, S. Zare, M. Onabajo, M. Li, Z. Zhou, T. Nan, X. Yang, M. Liu, K. Mahalingam, B. M. Howe, J. G. Jone, G. J Brown, N. X Sun, Microwave Symposium (IMS), 2014 IEEE MTT-S International, 1-4, 10.1109/MWSYM.2014.6848587.

${ }^{10}$ S. Marauska, R. Jahns, H. Greve, E. Quandt, R. Knochel, B. Wagner, J. Micromech. Microeng. 22, 065024 (2012).

11 M. Mohebbi, K. Ebnabbasi, C Vittoria, J. Appl. Phys. 113, 17C710 (2013).

12 K. Ebnabbasi, M. Mohebbi, C Vittoria, Appl. Phys. Lett. 101, 062406 (2012).

13 C. N. Johnson, P. D. D. Schwindt, M. Weisend, Phys Med Biol., 58, 6065 (2013)

14 R. Jahns, R. Knochel, H. Greve, E. Woltermann, E. Lage, E. Quandt, IEE trans. On Medical Measurement and Applications, DOI: $10.1109 / \mathrm{MeMeA} .2011 .5966676$.

15 K. Ebnabbasi, M. Mohebbi, C. Vittoria, J. Appl. Phys. 113, 17C707 (2013).

16 C. Vittoria, Magnetics, Dielectrics and Wave Propagation with MATLAB codes (CRC Press, Boca Raton, FL, 2010), pp. $167-227$.

${ }^{17}$ C. Vittoria, S. Somu, A. Widom, Phys. Rev. B. 89(13), 134413 (2014) 